\title{
Nanoscale
}

PAPER
View Article Online

View Journal / View Issue
Check for updates

Cite this: Nanoscale, 2019, 11, 6635

\section{Associative properties of poly(ethylene glycol)- poly(vinyl acetate) comb-like graft copolymers in water $\dagger$}

\author{
Marianna Mamusa, (iD a Paolo Tempesti, (iD a Arianna Bartolini, ta Emiliano Carretti, (iD a \\ Ahmad F. Ghobadi, ${ }^{b}$ Johan Smets, ${ }^{C}$ Yousef G. Aouad ${ }^{d}$ and Piero Baglioni (iD *a
}

Received 27th December 2018, Accepted 28th February 2019

DOI: $10.1039 / c 8 n r 10453 k$

rsc.li/nanoscale

\begin{abstract}
The self-assembly of amphiphilic graft copolymers is generally reported for polymer melts or polymers deposited onto surfaces, while a small number of cases deal with binary mixtures with water. We report on the associative properties of poly(ethylene glycol)-graft-poly(vinyl acetate) (PEG-g-PVAc) comb-like copolymers in water, demonstrating the existence of a percolative behaviour when increasing the PEG-g-PVAc content. Rheology, light- and small-angle X-ray scattering experiments, together with dissipative particle dynamics simulations, reveal a progressive transition from spherical polymer single-chain nanoparticles (SCNPs) towards hierarchically complex structures as the weight fraction of the polymer in water increases. The ability of PEG-g-PVAc to attain different nano- and microstructures is of great importance in numerous applications such as in the fields of cosmetics, detergency and drug delivery.
\end{abstract}

\section{Introduction}

Self-assembling amphiphilic copolymers in a block-selective solvent have gained considerable interest thanks to a broad range of potential applications, including but not limited to drug delivery, catalysis, and biomimetic materials. ${ }^{1,2}$ Although with some exceptions, ${ }^{3-6}$ most of the studies so far have focused on linear block copolymers, which have been found to associate into diverse morphologies. Spherical and cylindrical polymeric micelles are commonly obtained, ${ }^{7}$ especially through the assembly of di-block (A-B) and tri-block (A-B-A or

\footnotetext{
a Department of Chemistry "Ugo Schiff" and CSGI, University of Florence, Via della Lastruccia 3, Sesto Fiorentino, 50019 Florence, Italy.

E-mail: piero.baglioni@unifi.it; Tel: +39055 4573033

${ }^{b}$ Computational Chemistry, Modeling and Data Sciences, The Procter \& Gamble Company, Beckett Rd. 8611, West Chester, OH 45069, USA

${ }^{c}$ The Procter \& Gamble Company, Temselaan 100, 1853 Strombeek Bever, Belgium ${ }^{d}$ The Procter \& Gamble Company, Winton Hill Technical Center, 6100 Center Hill, Cincinnati, $\mathrm{OH} 45224$, USA

$\dagger$ Electronic supplementary information (ESI) available: Flow curves of PEG- $g$ $\mathrm{PVAc} / \mathrm{H}_{2} \mathrm{O}$ binary systems (Fig. SI1); amplitude sweep of PEG- $g$-PVAc $/ \mathrm{H}_{2} \mathrm{O}$ binary systems (Fig. SI2); frequency sweep of PEG- $g$-PVAc/ $\mathrm{H}_{2} \mathrm{O}$ binary systems (Fig. SI3); calculations for $\mathrm{H}_{2} \mathrm{O} / \mathrm{EO}$ molecular ratio in PEG- $g$-PVAc $60 \mathrm{wt} \%$; DSC thermograms of PEG- $g$-PVAc $/ \mathrm{H}_{2} \mathrm{O}$ binary systems (Fig. SI4); Summary of SAXS data fitting results (Table S1); SAXS patterns for PEG- $g$-PVAc $80 \mathrm{wt} \%$ and $90 \mathrm{wt} \%$ in water (Fig. SI5); SAXS curves of PEG- $g$-PVAc/ $\mathrm{H}_{2} \mathrm{O}$ binary systems at different temperatures (Fig. SI6); DPD simulation protocol; DPD simulation boxes (Table S3). See DOI: $10.1039 / \mathrm{c} 8 \mathrm{nr} 10453 \mathrm{k}$

\$Present address: Hoffmann Eitle S.r.l., Piazza Sigmund Freud 1, 20154 Milan, Italy.
}

B-A-B) amphiphilic copolymers. Depending on the arrangement of the polymer blocks and the derived interparticle potential, phenomena such as percolation, gelation, and glass transitions have been observed. ${ }^{8-10}$

Amphiphilic non-linear graft copolymers with chemically different backbone and side chains are attractive candidates for several applications, for example in drug delivery, food systems, and other technological applications. ${ }^{11-17}$ Their specific architecture is known to determine fundamentally different topology, properties, and solution behaviour with respect to linear block copolymers. ${ }^{18}$ Amphiphilic graft copolymers have been investigated less extensively than their linear counterparts, due to the only recent development of controlled polymerization methods such as controlled/living radical polymerization, "grafting to" and "grafting from" techniques. ${ }^{19-22}$ The solution behaviour of comb-like graft copolymers is influenced by the hydrophilic/hydrophobic features of the backbone and side chains: ${ }^{19,23}$ under poor solvent conditions for the grafts, the individual polymer molecules are subjected to attraction forces that lead to collapse and macroscopic phase separation; conversely, in the case of soluble grafts and collapsing backbones, intramolecular aggregates are formed and stabilized against aggregation by the repulsive forces between the side chain coronas. From several studies, graft copolymers have been found to display a variety of selfassembled aggregates in water or organic solvents with diverse morphologies and structures, ${ }^{24-27}$ the most exotic of which include petal-like micelles, spindle-like micelles, wormlike micelles, chiral helices, and snowflake-like suprastructures. ${ }^{28}$ 


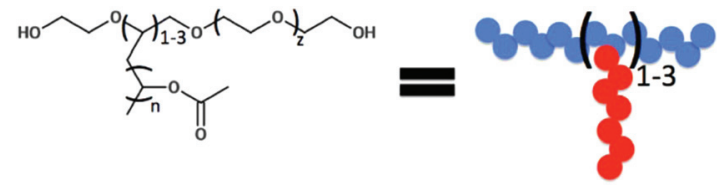

Scheme 1 Chemical structure of PEG-g-PVAc (left). Pictorial model of the PEG- $g$-PVAc structure (right). Subscripts $z$ and $n$ are the numbers of repetitive units of, respectively, ethylene oxide and vinyl acetate.

The self-assembly behaviour and the type of nanostructure morphology of graft copolymer solutions can also be tuned by playing with other factors such as grafting density, ${ }^{29,30}$ and crystallization in the case of semi-crystalline block copolymers (where one block is crystallizable and the other is amorphous). ${ }^{31}$

Among all amphiphilic graft copolymer molecules of interest, poly(ethylene glycol)-graft-poly(vinyl acetate) (PEG-g-PVAc) polymers exhibiting a low degree of grafting $(<5$ PVAc chains per 100 EG units, see Scheme 1) have found application in laundry detergent and cleaning compositions for their role as cleaning boosters. ${ }^{32,33}$ Their amphiphilicity is given by the fact that PEG is more hydrophilic than PVAc. Recent studies by our group highlighted that low-grafted PEG- $g$-PVAc can undergo self-folding into single-chain nanoparticles (SCNPs) when in water at concentrations below $10 \% \mathrm{w} / \mathrm{w}$, and they are able to introduce hydrophobic molecules into the PVAc core. ${ }^{34}$ The most interesting aspect here is that the SCNPs are formed spontaneously thanks to the amphiphilic properties of the copolymer, with no need for cross-linkers or external stimulibased strategies to drive the collapse and folding of one of the blocks. $^{35}$

Given the peculiar structures found in PEG- $g$-PVAc aqueous solutions at relatively low polymer concentrations, in the present work we expand the investigation in the $10-90 \% \mathrm{w} / \mathrm{w}$ concentration range and characterize the polymer's structural polymorphism by thermal, rheological and structural analyses, together with molecular calculations via Dissipative Particle Dynamics. In particular, a detailed description of the polymer chain arrangement when increasing the concentration is disclosed. Considering the biocompatibility of their block constituents, ${ }^{36}$ the results reported in this paper expand the knowledge on concentrated copolymer aqueous solutions, thus broadening their potential applications in systems involving biological molecules, such as drug delivery and tissue engineering.

\section{Results \& discussion}

Visual inspection of PEG- $g$-PVAc/water samples revealed that all specimens were isotropic and characterized by growing viscosity with increasing polymer content; in particular, polymer contents above $70 \%$ led to a very stiff consistency. Rheological tests were carried out both in the rotational mode and in the oscillation mode to understand the effect of the polymer concentration on the mechanical behaviour of the PEG-g-PVAc/ water systems. As indicated in Fig. SI1 (ESI $\dagger$ ), all the systems containing an amount of polymer lower than $10 \%$ are characterized by a Newtonian behaviour in the instrumentally accessible shear stress $(\sigma)$ range. At higher concentrations, a strong increase in the viscosity $\eta$ is observed along with a change in the profile of the flow curves: this indicates a non-Newtonian behaviour induced by the structuration of the system upon increasing the polymer content. In particular, the flow curves of the systems containing a polymer amount higher than $10 \%$ are characterized by the presence of a Newtonian region at low $\sigma$ (the corresponding $\eta$ value is the zero-shear viscosity, $\eta_{0}$ ) and by a critical value of $\sigma$ above which a shear-thinning is observed due to the disentanglement of the 3-D network formed by PEG- $g$-PVAc molecules. Fig. 1a reports the trend of $\eta_{0}$ as a function of the polymer content. The strong increase of the $\eta_{0}$ value upon increasing the polymer concentration up to $20 \%$ indicates a gradual structuration of the SNCPs into a more complex structure.

The viscoelastic behaviour of the PEG- $g$-PVAc/water binary system was also investigated, with specific attention to the dependence of the elastic and viscous moduli $\left(G^{\prime}\right.$ and $G^{\prime \prime}$, respectively) on the frequency of the applied shear stress. In particular, frequency sweep measurements were carried out in the linear viscoelastic regime previously measured through amplitude sweep tests (see the Experimental section and Fig. SI2, ESI $\dagger$ ); on the basis of these tests, the oscillation strain used for all the frequency sweep measurements was equal to $1 \%$. The profiles of the frequency sweeps (see Fig. SI3, ESI $\dagger$ ) indicate that all the investigated systems, independently of the polymer content, are characterized by a liquid-like behaviour as indicated by the trend of the $G^{\prime \prime}$ value that is always higher than $G^{\prime}$ all over the investigated frequency range. The drastic increase of the $G^{\prime}$ value (measured at $\omega=1 \mathrm{~Hz}$ ) upon increasing the polymer content above $20 \%$, as shown in Fig. 1a, is a further confirmation that above this threshold a structuration of the system occurs.

Fig. 1b shows the DSC thermograms obtained for all PEG- $g$ PVAc/water systems and the pure polymer. Due to the significant differences between the heat amount involved in the fusion of bulk water and in the polymer's transitions, individual curves for each sample are reported in Fig. SI4 in the ESI $\dagger$ for the sake of clarity. The pure polymer shows a glass transition at $T_{\mathrm{g}}=-34{ }^{\circ} \mathrm{C}$, followed by a melting transition $\left(T_{\mathrm{m}}\right)$ at $40{ }^{\circ} \mathrm{C}$, which is typically found in PEG chains. ${ }^{37}$ Indeed, PEG is known to crystallize in a monoclinic unit cell, with the macromolecules assuming a helical conformation consisting of seven monomer units per identity period of $1.9 \mathrm{~nm} .{ }^{38}$ Adding water up to $40 \%$ in weight suppresses both the $T_{\mathrm{g}}$ and the $T_{\mathrm{m}}$ (see Fig. SI4 in the ESI $\dagger$ for clarity). Samples containing 50\% to $10 \%$ polymer show an endothermic peak centred around $0{ }^{\circ} \mathrm{C}$, which can be related to the fusion of bulk water in the samples since no endothermic transitions are found for pure PEG- $g$-PVAc around $0{ }^{\circ} \mathrm{C}$. From simple calculations (see the $\mathrm{ESI}^{\dagger}$ ), it can be found that this polymer can bind up to 4 water molecules per EO unit, in very good agreement with typical values found in the literature for PEG. ${ }^{37,39}$ 

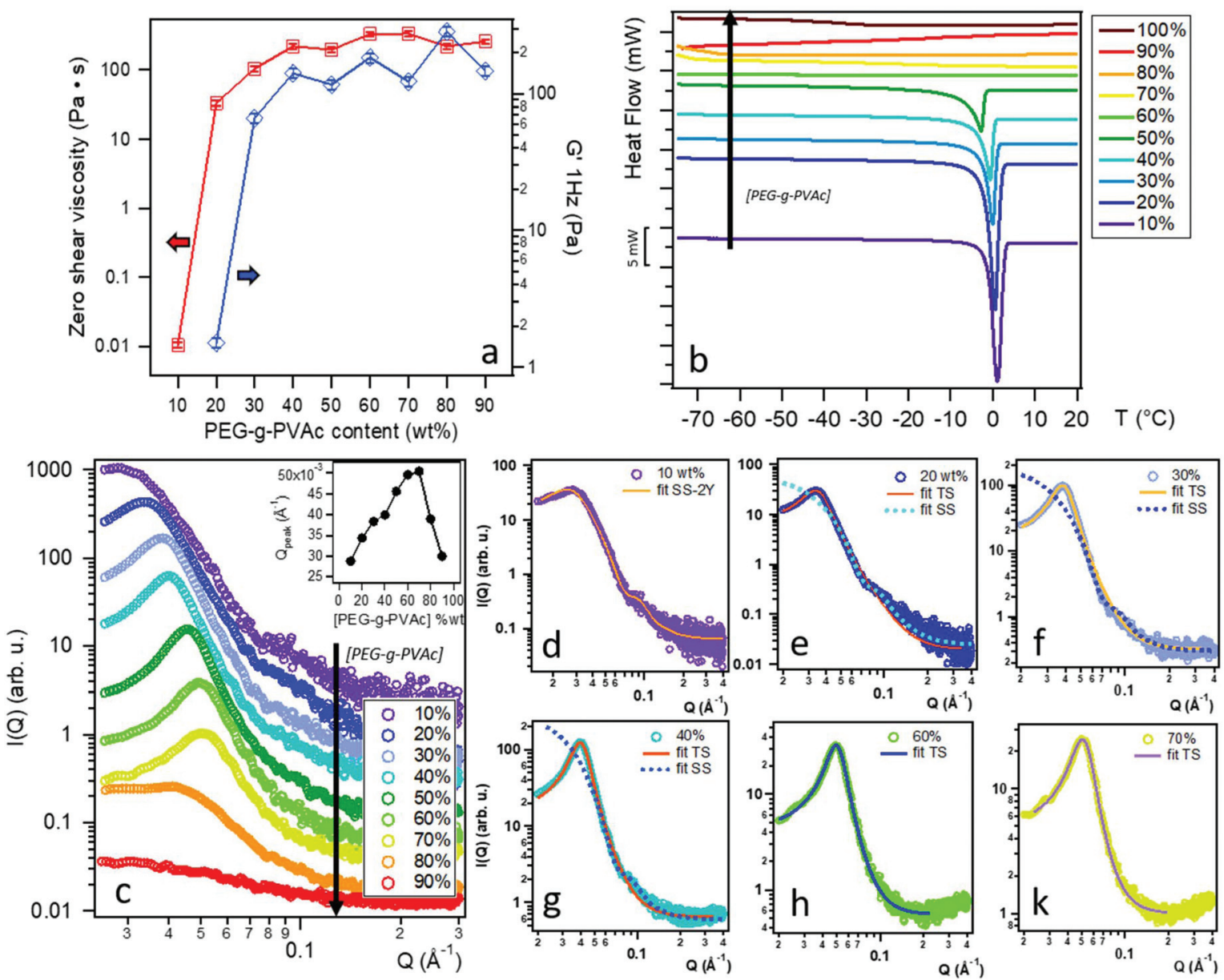

Fig. 1 (a) Trend of the $\eta_{0}$ values (red line with square markers) and of the $G^{\prime}$ measured at $\omega=1 \mathrm{~Hz}$ (blue line with diamond markers) as a function of PEG- $g$-PVAc content. Arrows point to the relevant $Y$ axis for each curve. (b) Thermograms obtained in the heating mode (exotherm up) for PEG- $g$ PVAc/water systems with different concentrations as indicated in the figure legend; curves have been offset along the Heat Flow axis for presentation purposes. (c) SAXS patterns obtained for PEG-g-PVAc/water systems with different concentrations as indicated in the figure legend. Curves have been offset along the $I(Q)$ axis for clarity of presentation. Inset: Trend of the peak maximum position $\left(Q_{\text {peak, }} \AA^{-1}\right)$ against polymer concentration. $(\mathrm{d}-\mathrm{k})$ Fit curves for some of the samples in panel (c); for each panel, the model used is indicated in the legend: SS-2Y (Schulz Spheres with twoYukawa interaction potential), SS (Schulz Spheres), TS (Teubner-Strey); more details can be found in the text.

Panel $\mathrm{c}$ in Fig. 1 shows the SAXS patterns obtained at $25^{\circ} \mathrm{C}$ for all the binary samples in the study. At a first analysis, the curves are dominated by an increasingly more pronounced correlation peak in the low- $Q$ region, which shifts to higher $Q$ values as the polymer concentration increases, and then this tendency is inversed after a critical threshold (70\% polymer content) is reached. This trend is highlighted in the inset of Fig. 1c, where the peak's position, $Q_{\text {peak }}$, is plotted against polymer concentration, and it is consistent with a percolative phase behaviour in agreement with the rheological results, as demonstrated in the discussion that follows. In the curve pertaining to the $10 \%$ polymer sample, the $Q_{\text {peak }}$ value $\left(0.03 \AA^{-1}\right)$ translates into a real-space distance $d_{\mathrm{SAXS}}=2 \pi / Q_{\text {peak }}=21.7 \mathrm{~nm}$, which is in good agreement with the hydrodynamic diameter of PEG- $g$-PVAc single-chain nanoparticles as reported for highly diluted systems. ${ }^{34}$ Therefore, $d_{\text {SAXs }}$ is likely the centreto-centre distance between SCNPs whose outer hydration shells are interacting. The SAXS pattern can be fitted (Fig. 1d) using the combination of a form factor of spheres (eqn (1)) with a $5.8 \mathrm{~nm}$ topologic radius and 18\% Schulz polydispersity (calculated through eqn (2)):

$$
\begin{gathered}
P(Q)=\frac{A}{V}\left[\frac{3 V(\Delta \rho)(\sin (Q r)-Q r \cos (Q r))}{(Q r)^{3}}\right]^{2}+I_{\mathrm{bkg}} \\
f(r)=(z+1)^{z+1} x^{z} \frac{\exp [-(z+1) x]}{r_{\mathrm{avg}} \Gamma(z+1)}
\end{gathered}
$$

where $A$ is a scale factor, $\Delta \rho$ is the scattering length density (SLD) difference between the particles and the solvent, and $I_{\text {bkg }}$ is the background offset; $r_{\text {avg }}$ is the mean radius and $x=$ $r / r_{\text {avg }} \Gamma$ is the Gamma function; $z$ is related to the polydispersity, $p=\sigma / r_{\mathrm{avg}}$, by $z=1 /\left(p^{2}-1\right)$, and $\sigma^{2}$ is the variance of the distribution.

The structure factor signature, represented by the low- $Q$ peak and downturn of the $I(Q)$ curve, is correctly modelled by 
implementing a two-Yukawa interaction potential (in its reduced form in eqn (3)), ${ }^{40}$ where the Ornstein-Zernike equation is solved using the Mean Spherical Approximation (MSA) closure (eqn (4)): ${ }^{41}$

$$
\begin{gathered}
\frac{V(r)}{k_{\mathrm{B}} T}= \begin{cases}\infty, & (0<r<1) \\
-K_{1} \frac{e^{-Z_{1}(r-1)}}{r}-K_{2} \frac{e^{-Z_{2}(r-1)}}{r}, & (r>1)\end{cases} \\
\begin{cases}h(r)=-1, & (0<r<1) \\
c(r)=\frac{V(r)}{k_{\mathrm{B}} T}=-K_{1} \frac{e^{-Z_{1}(r-1)}}{r}-K_{2} \frac{e^{-Z_{2}(r-1)}}{r}, & (r>1)\end{cases}
\end{gathered}
$$

Here, $k_{\mathrm{B}}$ is Boltzmann's constant, $T$ is the absolute temperature, $K_{1}$ and $K_{2}$ are the strengths and $Z_{1}$ and $Z_{2}$ are the inverse ranges of, respectively, the attractive and the repulsive parts of the potential. The two-Yukawa potential has been found to be capable of simulating realistic potentials (such as DLVO and Lennard Jones) in colloidal systems of either charged or uncharged particles. ${ }^{42}$ In the present case, the fitting parameters yielded $K_{1}=1.1, Z_{1}=4.6, K_{2}=-1.1$, and $Z_{2}=3.6$ : the condition $Z_{1}>Z_{2}$ is consistent with short-range attractive and long-range repulsive interactions. ${ }^{42}$

As the PEG- $g$-PVAc concentration increases, the $Q_{\text {peak }}$ value also increases, indicating a gradual reduction of the interparticle distance. At $20 \%$ polymer, fitting the SAXS pattern with one model for the entire $Q$-range is no longer possible: as shown in Fig. 1e, the high-Q region of the curve is still well modeled by a form factor of spherical objects with a radius of $5.8 \mathrm{~nm}$ and a Schultz polydispersity of the radii $\sigma=0.18$. However, the low- $Q$ region is no longer adequately interpreted by a two-Yukawa potential: the correlation peak can only be fitted by employing a Teubner-Strey model ${ }^{43}$ (eqn (5)) for bicontinuous structures:

$$
I(Q)=\frac{1}{a_{2}+c_{1} Q^{2}+c_{2} Q^{4}}+\mathrm{bkg}
$$

with $a_{2}, c_{1}$ and $c_{2}$ being variable parameters, and $b k g$ the background offset, which allowed the calculation of the quasiperiodic repeat distance $d$ (eqn (6)) and the correlation length $\xi$ (the dispersion of $d$; eqn (7)): ${ }^{44}$

$$
\begin{aligned}
& \frac{d}{2 \pi}=\left[\frac{1}{2}\left(\frac{a_{2}}{c_{2}}\right)^{\frac{1}{2}}-\frac{c_{1}}{4 c_{2}}\right]^{-\frac{1}{2}} \\
& \xi=\left[\frac{1}{2}\left(\frac{a_{2}}{c_{2}}\right)^{\frac{1}{2}}+\frac{c_{1}}{4 c_{2}}\right]^{-\frac{1}{2}}
\end{aligned}
$$

The situation is similar for the samples with $30 \%$ (Fig. 1f), 40\% (Fig. 1g), and 50\% polymer content (not shown), where the Schulz-spheres form factor is again applied to the high- $Q$ region of the curves, with appropriate adjustments for the background offset and scale factor. At the same time, the Teubner-Strey fitting yields increasing values of thermal correlation length $\xi$ and decreasing values of repeat distance $d$ (reported in Table 1). A complete summary of the fitting results for all samples can be found in the ESI (Table S1†).
Table 1 Structural parameters obtained from Teubner-Strey fit results on samples with the PEG-g-PVAc content between $20 \%$ and $70 \%$. Error bars are given based on instrumental resolution

\begin{tabular}{llll}
\hline $\begin{array}{l}\text { PEG- } g \text {-PVAc } \\
(\mathrm{wt} \%)\end{array}$ & $\begin{array}{l}\text { Correlation length, } \\
\xi(\AA) \pm 0.6 \AA\end{array}$ & $\begin{array}{l}\text { Repeat distance, } \\
d(\AA) \pm 0.6 \AA\end{array}$ & $d / \xi$ \\
\hline 20 & 119 & 182 & 1.5 \\
30 & 135 & 163 & 1.2 \\
40 & 144 & 156 & 1.1 \\
50 & 143 & 137 & 1.0 \\
60 & 115 & 125 & 1.1 \\
70 & 94 & 122 & 1.3
\end{tabular}

This picture delineates the existence of a bicontinuous polymer network in equilibrium with discrete SCNPs. The decrease of $d / \xi$ values with polymer concentration indicates a growing degree of order in the system; ${ }^{45}$ this tendency is however reversed for $70 \%$ PEG- $g$-PVAc.

The existence of a three-dimensional polymer network in this concentration range is further corroborated by DLS measurements showing a bimodal relaxation in the intensity autocorrelation functions (Fig. 2). At 10\% polymer, the DLS curves recorded at $90^{\circ}$ (full line) and $130^{\circ}$ (dotted line) appear consistent with a single, fast (low $\tau$ ) intensity decay. However, measurements at $45^{\circ}$ (dashed line) evidence a coexistent slow (high $\tau$ ) relaxation mode. ${ }^{46}$ This becomes more evident at all angles as the polymer concentration increases: the intercept of the autocorrelation functions decreases progressively and the curves tend to shift to higher $\tau$ values, which translates into slower diffusive motions, consistent with increased viscosity
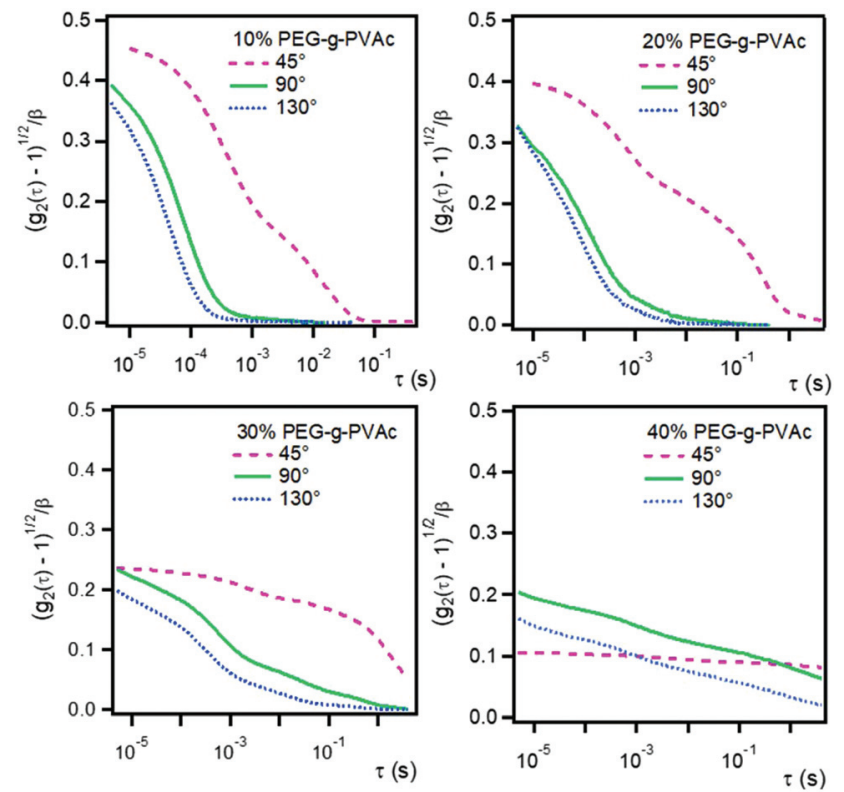

Fig. 2 Intensity autocorrelation functions of PEG-g-PVAc/water systems (ranging from $10 \%$ to $40 \%$ polymer weight) performed at different angles: $45^{\circ}$ (dashed pink lines), $90^{\circ}$ (solid green lines), and $130^{\circ}$ (dotted blue lines). A progressive transition from ergodic to nonergodic behaviour is evident when increasing polymer concentration, culminating with the almost flat autocorrelation curve for $40 \%$ polymer at $45^{\circ}$. 
and the formation of an extended polymer network. These facts clearly indicate that a continuous dynamic transition from ergodic to nonergodic (i.e., structurally arrested) behaviour occurs when increasing the polymer concentration from 10 to $40 \%$, in very good agreement with the rheological results (Fig. 1a). Measurements on the 50\% system (not shown) yielded noisy autocorrelation functions as the average scattering intensity of the sample decreased dramatically, and from $60 \%$ on the scattering intensity was too low to obtain any reliable autocorrelation function. Indeed, the scarce amount of water in the $60 \%$ polymer sample is only sufficient to grant hydration of the polymer chains, but it does not allow for the polymer to be dispersed in a continuous water medium; this fact is corroborated by the absence of a freezable water melting peak in the DSC curves for samples containing a polymer amount of $60 \%$ or more (Fig. 1b). Also, this probably determines the absence of a net contrast between the refractive index of a dispersed and dispersing phase, and it could be the reason for the scattering intensity of these samples becoming negligible, thus resulting in unfeasible DLS measurements.

The SAXS patterns (Fig. $1 \mathrm{~h}$ and $\mathrm{k}$ ) at $60 \%$ and $70 \%$ PEG- $g$ PVAc are correctly modeled using the Teubner-Strey function for the entire $Q$-range, indicating that the system is now composed entirely of a bicontinuous polymer network. The correlation distance $d_{\text {SAXs }}$ reaches here its minimum value, $12.6 \mathrm{~nm}$. At $80 \%$ polymer content, the correlation peak shifts back to $Q=0.047 \AA^{-1}\left(d_{\text {SAXs }}=13.4 \mathrm{~nm}\right)$, suggesting a reorganization of the polymer network. A closer look at the $80 \%$ sample (see ESI, Fig. SI5 in the ESI $\dagger$ ) shows that the correlation peak intensity is very low, the low- $Q$ trend is remindful of the $10 \%$ sample, and so is the $Q^{-4}$ decay law at high $Q$ values. These details may let us speculate on a sort of "structural inversion", with spherical water pockets surrounded by the PEG portion of the block copolymer, while the PVAc grafts form a continuous phase. At $90 \%$ polymer, the scattering intensity is low, and a correlation peak is no longer present. Despite the high polymer concentration, the sample remains amorphous since no Bragg peaks are present.

The PEG-g-PVAc/water systems were also investigated by SAXS upon increasing temperature (see ESI, Fig. SI6†). At 10\% polymer, a transition from spherical to elongated objects is observed, as inferred from the $Q^{-1}$ decay power law in the $0.01 \AA^{-1}<Q<0.02 \AA^{-1}$ range at $65^{\circ} \mathrm{C}$. This coincides with the polymer's cloud point as determined in previous work. ${ }^{34}$ The $20 \%$ sample does not show such a transition, as the SAXS pattern at $65{ }^{\circ} \mathrm{C}$ is consistent with spherical objects and the structure factor signature is lost; this seems to suggest that the interactions leading to the formation of the bicontinuous structure are weak and easily disrupted at high temperatures. However, at higher polymer concentrations (ESI, Fig. SI6C/E $\dagger$ ), the structure factor is dominant even at $65{ }^{\circ} \mathrm{C}$ and the form factor is no longer visible, indicating that the network-forming interactions are not disrupted under these conditions of concentration and temperature.

To gain additional molecular-level insight, we performed molecular dynamics simulations of systems comprising varying concentrations of PEG- $g$-PVAc in water. Due to slow dynamics of polymers, a coarse-grained model is required to explore experimentally relevant time and length scales. We chose Dissipative Particle Dynamics (DPD) simulations, a technique successfully used for the simulation of self-assembled systems of, among others, amphiphilic graft copolymers, ${ }^{47-49}$ in which a purely repulsive interaction potential along with dissipative and random forces is used to describe molecular interactions. $^{50}$ DPD simulations (see Fig. 3) on PEG- $g$-PVAc/ water systems are in excellent accordance with the results obtained from SAXS experiments and fittings. At 10\% PEG- $g$ -

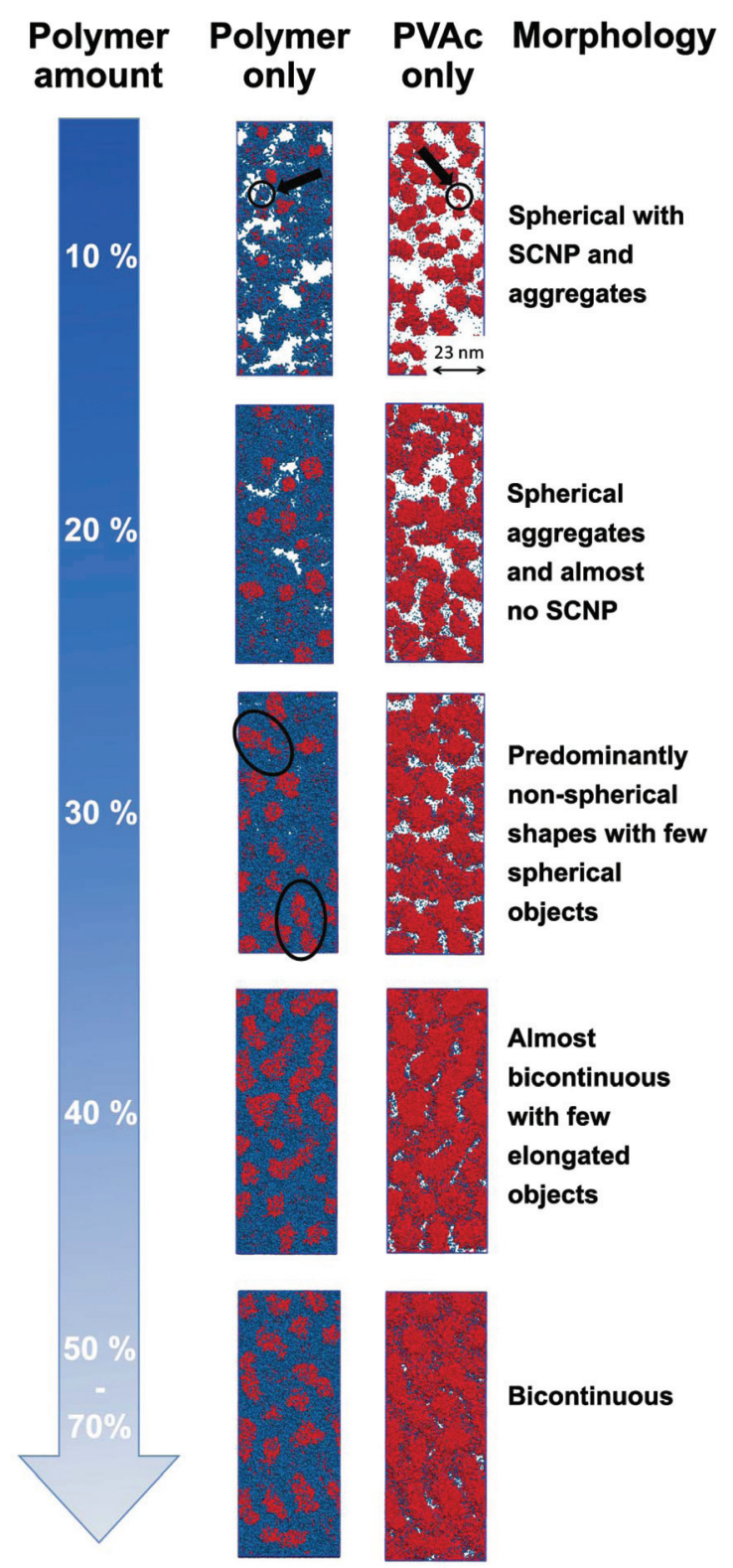

Fig. 3 Summary of observed equilibrium morphologies obtained by DPD simulations. $80 \%$ and $90 \%$ concentrations are reported in Table S3 in the ESI. $\dagger$ The blue and red colors correspond to PEG and PVAc chains, respectively. Arrows indicate PEG-g-PVAc SCNP, while black ellipsoids indicate elongated aggregates. 
PVAc concentration in water, the predominant morphology in solution is represented by spherical SCNPs and aggregates. In these spherical aggregates, the PEG moiety in the polymer enfolds the PVAc moiety to shield water/PVAc interactions and to lower the surface tension of the aggregates. Also, the spherical shape maximizes the access of PEG to bulk water, leading to an increase in the number of unique configurations that PEG can adopt in water and, hence, maximizing their entropy. Therefore, spherical objects are favorable from both the enthalpic and the entropic points of view. At $20 \%$, the weight fraction of spherical aggregates is increased at the expense of SCNPs. This aligns with our expectations in that increasing concentration of SCNPs eventually results in increasing interpenetration (or contact) of PEG moieties on separate SCNPs, which enhances the entropic cost of maintaining the SCNP structure.

Therefore, increasing polymer concentration encourages SCNPs to reorganize and merge to form larger aggregates. It is also important to note that the merging of SCNPs also increases PVAc/PVAc contact, and hence it benefits the overall free energy of the system via hydrophobic interactions. At polymer concentrations from $30 \%$ to $50 \%$, further aggregation between spherical aggregates results in the formation of nonregular elongated objects, and a progressive trend toward a bicontinuous structure is detected. The formation of a bicontinuous phase minimizes the free energy of the system by accommodating more hydrophobic-hydrophobic interactions and alleviating the lower free volume available to individual aggregates as the concentration of the polymer increases. From $60 \%$ to $70 \%$, the polymer is structured in a bicontinuous arrangement in very good accordance with SAXS experiments. In the system with $80 \%$ polymer (see Table S3 in the ESI $\dagger$ ), the PEG and water regions are overlapped, thus confirming the suggestion of a "structural inversion" assumed by SAXS data analysis. Finally, for $90 \%$ polymer content the water molecules are almost homogeneously dispersed in an amorphous polymer continuous phase with no relevant ordered structures (Table S3, ESI $\dagger$ ), as again confirmed by SAXS experiments.

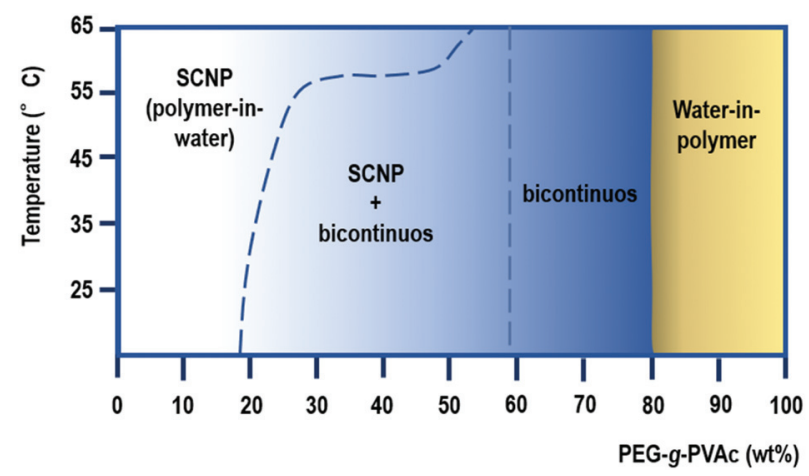

Fig. 4 Gibbs phase diagram for the PEG-g-PVAc/water binary system as a function of temperature and of polymer concentration. The color gradient in the $0-80 \%$ polymer range indicates the progressive transition from SCNPs to the bicontinuous phase.
It is important to remark that the DPD simulations reported here are in very good agreement with SAXS experimental results despite not considering the polydispersity in the polymer's $M_{\mathrm{w}}$, grafting density, and PVAc graft locations along the PEG backbone during calculations. The data obtained thus far allow us, therefore, to draw the thermodynamic binary phase diagram for the PEG-g-PVAc/water system (Fig. 4).

\section{Conclusions}

Previous studies have shown that the here reported comb-like PEG- $g$-PVAc graft copolymer with low grafting density was capable of self-folding in water into single-chain polymer nanoparticles (SCNPs) in a concentration range up to $10 \% .^{34}$ The most intriguing aspect of these nanoparticles was the spontaneous formation by an intra-molecular self-assembly mechanism driven simply by the amphiphilic character of the copolymer, while most SCNPs described in the literature require the presence of either appropriate cross-linking agents, at specific points along the polymer segments, or the introduction of external stimuli like $\mathrm{pH}$ variation or UV irradiation to drive the self-folding process. ${ }^{35}$ In the present work, we extended the investigation of the binary system PEG-g-PVAc/ water to polymer concentrations in the 10-90 wt\% range, using rheological and scattering experiments that are fully in agreement with DPD simulations, demonstrating a progressive structuration of the SCNPs into hierarchically complex systems when increasing the polymer concentration. In particular, rheology studies and SAXS measurements revealed the formation of an extended 3D network above $20 \%$ polymer in water, with a SCNP/bicontinuous coexistence region, consisting in a percolative behavior. The presence of a bicontinuous network was further corroborated by the observation of two relaxation modes in dynamic light scattering experiments, and by DPD computer simulations, the latter hinting at the persistence of the single-chain nanoparticle building blocks even in the bicontinuous region. Modelling of SAXS data indicated that the system behaves like a two-Yukawa fluid, ${ }^{40}$ with the polymer nanoparticles interacting via long-range repulsive interactions and a short-range attraction: the SCNPs therefore start forming clusters above a critical concentration threshold, giving rise to a bicontinuous network of increasing structural order with polymer concentration. The correlation length reaches its minimum value in correspondence with the SCNP topologic diameter before a structural inversion is observed at $80 \%$ polymer, once again strongly supporting the concept that the SCNPs themselves remain as the very building blocks of this extended polymer network. To the best of our knowledge, a full phase diagram of a comb-like copolymer showing these properties has not yet been described. Therefore, this work not only adds to the growing bulk of literature on the physicalchemical properties of comb-like copolymer systems, which are still poorly investigated, but it also reveals the possibility of fine-tuning the choice of the constituting polymer blocks to obtain functional structures which maintain the properties of 
their elementary units even as extended, concentrated networks are formed. Ideally, these systems can be used to encapsulate the target substances (perfumes, flavors, drugs etc.) or even a multitude of these simultaneously in SCNPs, then concentrated, and eventually diluted again at the time of application, without losing the properties of the building blocks and maintaining the individual functionality of each unit, paving the way to the exploitation of these biodegradable polymers in several industrial and clinical applications.

\section{Experimental}

\section{Materials}

PEG- $g$-PVAc was synthesized as previously described. ${ }^{34}$ The polymer is characterized by a PEG/PVAc weight ratio of $40 / 60$, $M_{\mathrm{n}}=13.1 \mathrm{kDa}, \mathrm{PDI}=2.1$, and degree of branching of $1-2 \%$. Given the polymer's $M_{\mathrm{n}}$ and PEG/PVAc weight ratio, the number of ethylene oxide $(z)$ and vinyl acetate $(n)$ repeating units is, respectively, $\sim 130$ and $\sim 80$.

Water used in this work was Milli-Q grade $(18.2 \mathrm{M} \Omega \mathrm{cm}$ at $\left.25^{\circ} \mathrm{C}\right)$.

\section{Sample preparation}

Samples with polymer contents ranging between $10 \%$ and $90 \% \mathrm{w} / \mathrm{w}$ in distilled water were prepared to study PEG- $g$-PVAc/ water binary phase behavior. The polymer was molten at $50{ }^{\circ} \mathrm{C}$ for ease of manipulation; upon addition of the appropriate amount of water, samples were vortexed until homogenization and stabilized at $25^{\circ} \mathrm{C}$ in an oven for 14 days. In the present work, concentrations are always expressed as weight percent unless specified.

\section{Rheology}

Rheological measurements were carried out on an Anton Paar UDS 200 rheometer that works in the controlled shear stress mode, using a plate-plate geometry (Flat Plate $50 \mathrm{~mm}$ diameter) and a Peltier system for temperature control. The gap between the plates at zero radial position was always maintained equal to $300 \mu \mathrm{m}$. The cell was closed by lowering the head to the measuring position in the $z$-axis force-controlled mode; the maximum value of the normal force while closing the cell was $0.5 \mathrm{~N}$. Before starting the experiments, the samples were left to equilibrate for $30 \mathrm{~min}$ at $25^{\circ} \mathrm{C}$. Frequency sweep measurements were carried out within the linear viscoelastic range (the strain is equal to $1 \%$ ), determined by means of an amplitude sweep test (see Fig. SI1 and SI $2 \dagger$ ). The storage and loss moduli $\left(G^{\prime}\right.$ and $\left.G^{\prime \prime}\right)$ were measured over the frequency range of $0.001-100 \mathrm{~Hz}$. The flow curves were collected by measuring the viscosity values under the application of a shear stress $(\sigma)$ logarithmic ramp in the range of $1-10^{4} \mathrm{~Pa}$. The zero-shear viscosity, $\eta_{0}$, is defined as the viscosity value at low shear stress $\sigma$ where the fluids are characterized by a Newtonian behavior (low Newtonian region, Fig. SI3†).

\section{Differential scanning calorimetry (DSC)}

DSC analysis was performed with a Q2000 DSC, TA Instruments (New Castle, USA). Roughly $30 \mathrm{mg}$ of each sample were placed in steel pans and analyzed in the heating mode, between $-80^{\circ} \mathrm{C}$ and $50^{\circ} \mathrm{C}$, at a scan rate of $0.5^{\circ} \mathrm{C} \mathrm{min}^{-1}$. The Free Water Content (FWC) was calculated as follows:

$$
\mathrm{FWC}=\left(\frac{\Delta H_{\mathrm{f}, \text { sample }} \cdot m_{\text {sample }}}{\Delta H_{\mathrm{f}, \mathrm{w}}}\right) \times \frac{100}{m_{\mathrm{w}}}
$$

where $\Delta H_{\mathrm{f}, \text { sample }}$ is the enthalpy of fusion of water in the samples in $\mathrm{J} \mathrm{g}^{-1}, m_{\text {sample }}$ is the weight of the sample in $\mathrm{g}$, $m_{\mathrm{w}}$ is the nominal amount of water in the sample in $\mathrm{g}$, and $\Delta H_{\mathrm{f}, \mathrm{w}}$ is the enthalpy of fusion of pure water. $\Delta H_{\mathrm{f} \text {,sample }}$ and $\Delta H_{\mathrm{f}, \mathrm{w}}$ were obtained by integrating thermal peaks between $-20{ }^{\circ} \mathrm{C}$ and $+5{ }^{\circ} \mathrm{C}$ of samples and Milli-Q grade water, respectively.

\section{Dynamic light scattering (DLS)}

DLS measurements were performed on a Brookhaven BI9000AT digital autocorrelator, equipped with a green laser ( $\lambda=532 \mathrm{~nm}$; Torus, mpc3000, LaserQuantum, UK) and an APD detector placed at $45^{\circ}, 90^{\circ}$, and $135^{\circ}$. Samples were enclosed in a glass test-tube and immersed in a thermostated VAT, filled with decahydronaphtalene as a glass refraction index matching liquid. Experiments were performed at $25^{\circ} \mathrm{C}$. Intensity-normalized autocorrelation functions reported here are the average of 10 independent measurements, each acquired for 15 minutes with varying scattering volume, for each angle. The ordinates report the measured time autocorrelation functions as $C(\tau)=\left(g_{2}(\tau)-1\right)^{1 / 2} / \beta$ (where $\beta$ is the spatial coherence factor), expressed according to the Siegert relation.

\section{Dissipative particle dynamics (DPD) simulation protocols}

All DPD simulations were performed using an in-house procedure. To parameterize our DPD model, first the molecular structures were optimized by $a b$ initio calculations. Then, gas phase atomistic simulations were performed to parameterize molecular bond and angle rigidity. Finally, continuum solvation models were used to parameterize the DPD intermolecular interactions. The parameterization workflow is further explained in the ESI. $\dagger$

\section{Small-angle X-ray scattering (SAXS)}

In-house SAXS analysis was performed on a Kratky camera system (HECUS). The incident beam was $\mathrm{CuK}_{\alpha}$ radiation $(\lambda=1.542 \AA)$ produced by a sealed-tube generator (Seifert ID-303) operating at $2 \mathrm{~kW}$; the $\mathrm{CuK}_{\beta}$ radiation was removed thanks to a $10 \mu \mathrm{m}$ thick Ni filter. The detector (OED $50 \mathrm{M}$ ) contained 1024 channels of width $54 \mu \mathrm{m}$, and the sample-todetector distance was $275 \mathrm{~mm}$. The available $Q$-range was 0.01-0.55 $\AA^{-1}$. Samples were inserted into either a quartz Mark capillary ( $2 \mathrm{~mm}$ diameter) or a paste sample holder, depending on their viscosity, and the cells were kept under vacuum during the experiment. Measurements were performed in the 
25-65 ${ }^{\circ} \mathrm{C}$ temperature range (temperature controlled by a Peltier element with $\pm 0.1{ }^{\circ} \mathrm{C}$ accuracy). In a small-angle X-ray experiment, the scattered intensity $I(Q)$ is described by the general relationship:

$$
I(Q) \propto P(Q) S(Q)
$$

where $Q$ is the modulus of the scattering vector $(4 \pi / \lambda \sin \theta$, with $2 \theta$ the angle between the incident beam and the scattered beam), $P(Q)$ is the form factor and $S(Q)$ is the structure factor.

\section{Conflicts of interest}

There are no conflicts to declare.

\section{Acknowledgements}

The authors acknowledge financial support from Procter \& Gamble Co and from Consorzio Interuniversitario per lo Sviluppo dei Sistemi a Grande Interfase (CSGI).

\section{Notes and references}

1 Z. Zhang, R. Ma and L. Shi, Acc. Chem. Res., 2014, 47, 14261437.

2 Y. Wang, L. Hosta-Rigau, H. Lomas and F. Caruso, Phys. Chem. Chem. Phys., 2011, 13, 4782.

3 X. Ye, T. Shi, Z. Lu, C. Zhang, Z. Sun and L. An, Macromolecules, 2005, 38, 8853-8857.

4 A. Durand and D. Hourdet, Polymer, 1999, 40, 4941-4951.

5 F. L. Beyer, S. P. Gido, C. Büschl, H. Iatrou, D. Uhrig, J. W. Mays, M. Y. Chang, B. A. Garetz, N. P. Balsara, N. B. Tan and N. Hadjichristidis, Macromolecules, 2000, 33, 2039-2048.

6 L. Zhang, J. Lin and S. Lin, J. Phys. Chem. B, 2008, 112, 9720-9728.

7 Y. Mai and A. Eisenberg, Chem. Soc. Rev., 2012, 41, 5969.

8 S. H. Chen, C. Liao, E. Fratini, P. Baglioni and F. Mallamace, Colloids Surf., A, 2001, 183-185, 95-111.

9 W.-R. Chen, S.-H. Chen and F. Mallamace, Phys. Rev. E: Stat., Nonlinear, Soft Matter Phys., 2002, 66, 021403.

10 V. Hugouvieux, M. A. V. Axelos and M. Kolb, Soft Matter, 2011, 7, 2580.

11 Y. Sun, X. Yan, T. Yuan, J. Liang, Y. Fan, Z. Gu and X. Zhang, Biomaterials, 2010, 31, 7124-7131.

12 R. Gref, J. Rodrigues and P. Couvreur, Macromolecules, 2002, 35, 9861-9867.

13 S. Seslija, P. Spasojević, V. Panić, M. Dobrzyńska-Mizera, B. Immirzi, J. Stevanović and I. Popović, Int. J. Biol. Macromol., 2018, 113, 924-932.

14 S. Alam, H. Kalita, O. Kudina, A. Popadyuk, B. J. Chisholm and A. Voronov, ACS Sustainable Chem. Eng., 2013, 1, 19-22.

15 A. Najer, D. Wu, M. G. Nussbaumer, G. Schwertz, A. Schwab, M. C. Witschel, A. Schäfer, F. Diederich,
M. Rottmann, C. G. Palivan, H.-P. Beck and W. Meier, Nanoscale, 2016, 8, 14858-14869.

16 D. J. Kim, S. J. Kim, D. K. Roh and J. H. Kim, Phys. Chem. Chem. Phys., 2013, 15, 7345.

17 E. Guzmán, F. Ortega, M. G. Prolongo, V. M. Starov and R. G. Rubio, Phys. Chem. Chem. Phys., 2011, 13, 16416.

18 A. Ding, J. Xu, G. Gu, G. Lu and X. Huang, Sci. Rep., 2017, 7, 12601.

19 O. V. Borisov and E. B. Zhulina, Macromolecules, 2005, 38, 2506-2514.

20 P. Raffa, D. A. Z. Wever, F. Picchioni and A. A. Broekhuis, Chem. Rev., 2015, 115, 8504-8563.

21 Y. Xia, B. D. Olsen, J. A. Kornfield and R. H. Grubbs, J. Am. Chem. Soc., 2009, 131, 18525-18532.

22 G. Cavallaro, M. Licciardi, M. D. Stefano, G. Pitarresi and G. Giammona, Macromolecules, 2009, 42, 3247-3257.

23 Z. Posel, M. Svoboda, Z. Limpouchová, M. Lísal and K. Procházka, Phys. Chem. Chem. Phys., 2018, 20, 65336547.

24 Y. Li, Y. Zhang, D. Yang, Y. Li, J. Hu, C. Feng, S. Zhai, G. Lu and X. Huang, Macromolecules, 2010, 43, 262-270.

25 B. Xu, G. Gu, C. Feng, X. Jiang, J. Hu, G. Lu, S. Zhang and X. Huang, Polym. Chem., 2016, 7, 613-624.

26 C. Cai, J. Lin, T. Chen and X. Tian, Langmuir, 2010, 26, 2791-2797.

27 B. Li, G. Chen, F. Meng, T. Li, J. Yue, X. Jing and Y. Huang, Polym. Chem., 2012, 3, 2421.

28 A. L. Parry, P. H. H. Bomans, S. J. Holder, N. A. J. M. Sommerdijk and S. C. G. Biagini, Angew. Chem., Int. Ed., 2008, 47, 8859-8862.

29 E. B. Zhulina and T. A. Vilgis, Macromolecules, 1995, 28, 1008-1015.

30 H. Chen and E. Ruckenstein, Soft Matter, 2012, 8, 13271333.

31 A. Presa Soto, J. B. Gilroy, M. A. Winnik and I. Manners, Angew. Chem., Int. Ed., 2010, 49, 8220-8223.

32 D. Boeckh, L. Herrera Taboada, A. Kavarnou-Seiler, G. Konrad, B. Reinhard, A. L. Casado Dominguez, J. Danziger and F. Hulskotter, Cleaning Compositions with Amphiphilic Graft Polymers Based on Polyalkylene Oxides and Vinyl Esters, PCT/EP2007/055198, 2007.

33 Y. G. Aouad, J. Smets, L. A. Zannoni, P. Baglioni, P. Tempesti and A. Bartolini, Benefit agent containing delivery particle, WO2018052990A1, 2018.

34 A. Bartolini, P. Tempesti, C. Resta, D. Berti, J. Smets, Y. G. Aouad and P. Baglioni, Phys. Chem. Chem. Phys., 2017, 19, 4553-4559.

35 M. K. Aiertza, I. Odriozola, G. Cabañero, H.-J. Grande and I. Loinaz, Cell. Mol. Life Sci., 2012, 69, 337-346.

36 N. Bailly, M. Thomas and B. Klumperman, Biomacromolecules, 2012, 13, 4109-4117.

37 L. Huang and K. Nishinari, J. Polym. Sci., Part B: Polym. Phys., 2001, 39, 496-506.

38 M. D. Mihaylova, V. P. Krestev, M. N. Kresteva, A. Amzil and I. V. Berlinova, Eur. Polym. J., 2001, 37, 233-239. 
39 T. Hatakeyma, H. Kasuga, M. Tanaka and H. Hatakeyama, Thermochim. Acta, 2007, 465, 59-66.

40 Y. Liu, W.-R. Chen and S.-H. Chen, J. Chem. Phys., 2005, 122, 044507.

41 J. S. Høye and L. Blum, J. Stat. Phys., 1977, 16, 399-413.

42 S.-H. Chen, M. Broccio, Y. Liu, E. Fratini and P. Baglioni, J. Appl. Crystallogr., 2007, 40, s321-s326.

43 M. Teubner and R. Strey, J. Chem. Phys., 1987, 87, 31953200.

44 K.-V. Schubert, R. Strey, S. R. Kline and E. W. Kaler, J. Chem. Phys., 1994, 101, 5343-5355.
45 H. Leitão, M. M. Telo da Gama and R. Strey, J. Chem. Phys., 1998, 108, 4189-4198.

46 J. Li, T. Ngai and C. Wu, Polym. J., 2010, 42, 609-625.

47 J. Xu, S. Sun, Z. Wang, S. Peng, S. Hu and L. Zhang, Phys. Chem. Chem. Phys., 2018, 20, 9460-9470.

48 J. Xia, D. Liu and C. Zhong, Phys. Chem. Chem. Phys., 2007, 9, 5267.

49 P. Xu, J. Lin and L. Zhang, Phys. Chem. Chem. Phys., 2018, 20, 15995-16004.

50 P. Español and P. B. Warren, J. Chem. Phys., 2017, 146, 150901. 